\title{
NOVEL BEAMFORMERS FOR MULTIPLE CORRELATED BRAIN SOURCE LOCALIZATION AND RECONSTRUCTION
}

\author{
Hung V. Dang, Kwong T. Ng* \\ New Mexico State University \\ Department of Electrical and Computer Engineering \\ P.O. Box 30001/MSC 3-O \\ Las Cruces, NM 88003
}

\author{
James K. Kroger \\ New Mexico State University \\ Department of Psychology \\ PO Box 30001/MSC 3452 \\ Las Cruces, NM 88003
}

\begin{abstract}
This paper introduces two novel beamforming algorithms, namely the Region Constrained and Multiple Correlated Source Model beamformers, designed to localize and to reconstruct highly correlated brain sources from noisy EEG data. Multiple correlated source simulations have been performed to evaluate the performance of the proposed algorithms, using a realistic $176 \times 240 \times 256$ finite difference head model. Our simulation results show that the Region Constrained-Multiple Correlated Source Model beamformer, obtained by combining the above two beamformers, allows us to localize three perfectly correlated brain sources with very high localization accuracy. Finally, the eigenspace version of this beamformer can be used to reconstruct three correlated brain source signals correctly from simulated noisy EEG data.
\end{abstract}

Index Terms - Beamformer, beamforming algorithm, EEG, FDM, forward model.

\section{INTRODUCTION}

Electroencephalography (EEG) inverse source imaging has been widely used to study brain functions and to monitor neural activities [1]. The recorded EEG signals have been used to localize and to reconstruct the brain sources [1], typically represented as electric current dipoles. Numerous inverse solution techniques have been studied in the past [1]. Each of these techniques relies on its own assumptions and constraints to localize the sources. Among them is the beamforming approach that has been explored as a possible way to improve the spatial accuracy of source imaging [2, 3, 4, 5, 6, 7]. A beamformer is essentially a spatial filter that can be applied to any location in the brain. By suppressing the effects of sources at all other places, a beamformer allows us to estimate the source at that particular spatial location from a segment of EEG signals.

* The research was supported in part by the Los Alamos National Laboratory and Army High Performance Computing Research Center.
There are two major types of beamforming algorithms: 1) scalar beamformers in which the dipole orientation is assumed to be fixed and estimated separately, and 2) vector beamformers that estimate the three dipole components simultaneously. Three major scalar beamformers were proposed in the past, namely the scalar minimum variance beamformer (S-MVB) [8], the scalar weight normalized minimum variance beamformer (S-WNMVB) and the scalar standardized minimum variance beamformer (S-SMVB) [9]. As for the vector beamformers, the most common one is the vector minimum variance beamformer or linearly constrained minimum variance beamformer (LCMV), which was first proposed in [2]. Later, the unit-noise power vector beamformer or vector Borgiotti-Kaplan beamformer (V-BKB) was introduced in [10], where it was shown to perform better than LCMV.

The above five algorithms will be hereafter referred to as the "regular" beamformers. Like other inverse algorithms, these beamformers have their own drawbacks [11] because they are based on the assumption that brain sources are temporally and spatially uncorrelated. In reality this assumption is seldom satisfied, which in turn leads to errors in source localization and reconstruction, especially when there is a large correlation between the sources or background interference.

To improve the performance of the above beamformers, a number of techniques with different additional constraints were developed. These can be classified into two main categories: 1) using additional temporal information of brain sources to further constrain the solution and 2) exploiting the spatial information of brain sources as the constraint. The specific approaches proposed are: 1) eigenspace beamformer [4]; 2) prewhitening beamforming [12]; 3) synthetic aperture magnetoencephalography (SAM) [3]; 4) null constrained beamformer [6]; 5) Backus-Gilbert resolution spread function-constrained and fMRI-guided spatial filter [11]; 6) dynamic imaging of coherent sources (DICS) [5]; 7) dualcore beamformer (DCB) [13].

The motivation of this work arises from the need for a better beamformer for localizing and reconstructing highly cor- 

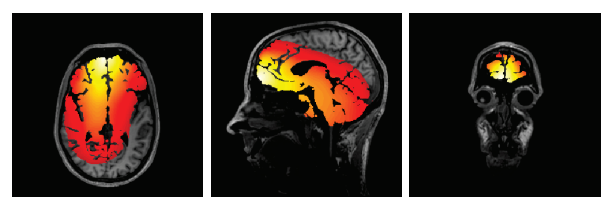

Fig. 1: The output tomographic maps of S-MVB
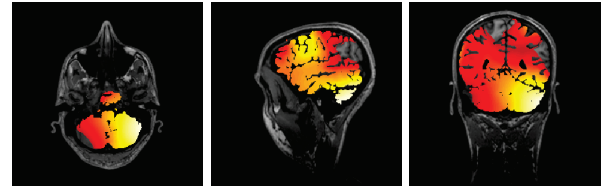

Fig. 2: The output tomographic maps of SAM

related brain sources. Here, we propose the multiple correlated source model and region constrained beamforming algorithms, which our simulation results show are promising in solving the highly correlated source problem.

The proposed beamforming algorithms are described in the next section. In the results section, the performance of the new algorithms are evaluated in more realistic conditions with 3 perfectly correlated brain sources and additive Gaussian white noise. The studies are carried out using a $176 \times 240 \times$ 256 head model with a resolution of $1 \mathrm{~mm} \times 1 \mathrm{~mm} \times 1 \mathrm{~mm}$ and 6 tissues, constructed from an anatomical MR image using the FMRIB Software Library (FSL). We have developed the Finite Difference Neuroelectromagnetic Head Modeling Software (FNS) to collect all the required forward solutions for an 128-electrode array.

In the following sections, lower case Greek letters represent scalars, lower bold case Roman letters represent column vectors, and upper bold case Greek or Roman letters represent matrices.

\section{NOVEL BEAMFORMING ALGORITHMS}

\subsection{Multiple correlated source model beamformer (MC- SMB)}

Assuming we have $K$ totally correlated sources, so the crosscorrelation between any two sources is perfect or $\frac{\mathbf{s}_{j}^{T} \mathbf{s}_{k}}{\mathbf{s}_{k}^{T} \mathbf{s}_{k}}=1$. The simulated EEG signals are computed as

$$
\begin{aligned}
\mathbf{m} & =\mathbf{g}_{1} \mathbf{s}_{1}+\ldots+\mathbf{g}_{K} \mathbf{s}_{K}+\mathbf{n} \\
& =\left(\sum_{k=1}^{K} \lambda_{k} \mathbf{g}_{k}\right) \mathbf{s}_{o}+\mathbf{n}
\end{aligned}
$$

where $\mathbf{m}$ are the EEG signals, $\mathbf{s}_{k}=\lambda_{k} \mathbf{s}_{0}$ represents the $k^{\text {th }}$ source, $\mathbf{g}_{k}=\mathbf{L}_{k} \mathbf{v}_{k}$ is the corresponding gain vector, with
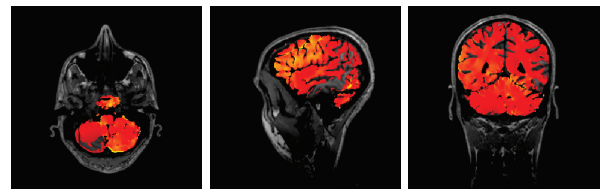

Fig. 3: The output tomographic map of LCMV
$\mathbf{L}_{k}$ and $\mathbf{v}_{k}$ corresponding to the lead field matrix and the source orientation, respectively. Here, $\mathbf{g}=\sum_{k=1}^{K} \lambda_{k} \mathbf{g}_{k}=$ $\left[\mathbf{L}_{1}, \ldots, \mathbf{L}_{K}\right]\left[\begin{array}{c}\lambda_{1} \mathbf{v}_{1} \\ \ldots \\ \lambda_{k} \mathbf{v}_{k}\end{array}\right]=\mathbf{L v}$ and the additive noise, $\mathbf{n}$, is

Gaussian white noise. The composite optimum orientation $\mathbf{v}$ for a scalar beamformer can be found by optimizing the output power or the output SNR of S-MVB, as described in [3]. This beamformer is the generalization of the dual-core beamformers, which has been recently introduced in [13].

\subsection{Region constrained (RC) beamformer}

For this beamformer the covariance matrix is modified as

$$
\mathbf{R}_{r}=\mathbf{R}+\beta \mathbf{S}+\gamma^{2} \mathbf{I}
$$

where $\alpha$ and $\beta$ are the scale factors, $\mathbf{S}=\mathbf{G} \mathbf{A G}^{T}, \mathbf{G}$ is the lead field matrix of the constrained region, $\mathbf{A}$ is the weight matrix which can be obtained according to the location of the constrained region ( 1 for nodes belonging to the region of interest and 0 otherwise), and $\gamma^{2}$ is the diagonal loading factor. The procedure to modify the covariance matrix is similar to what has been described in [11].

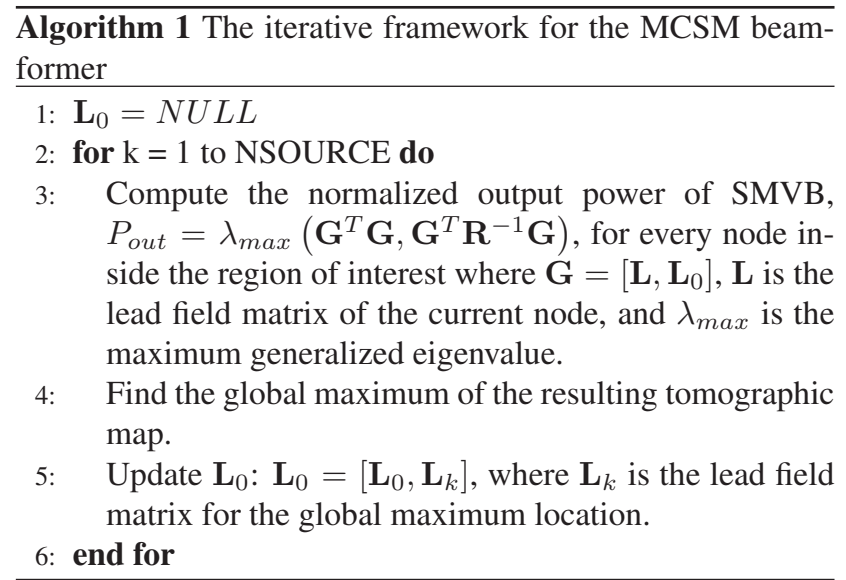

\section{RESULTS}

In this study, we simulate 3 brain sources to imitate the physiological distribution of spontaneous brain activity. The first two sources at $12 \mathrm{~Hz}$ are located in the frontal cortex and in intraparietal sulcus. The third source, also at $12 \mathrm{~Hz}$, is located in the sensorimotor hand area. The coherence between these 3 sources is 1 , so they are perfectly correlated, and amplitudes of the three sources are $3 \mu A, 5 \mu A$ and $1 \mu A$, respectively. To make the simulation more realistic, Gaussian white noise is added with $S N R=4 \mathrm{~dB}$.

Figure 1, 2 and 3 show the output of S-MVB, SAM and LCMV. We can see clearly that the tomographic maps of these 

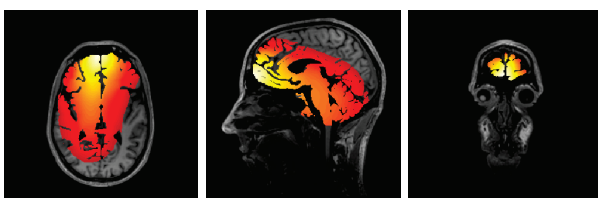

Step 1
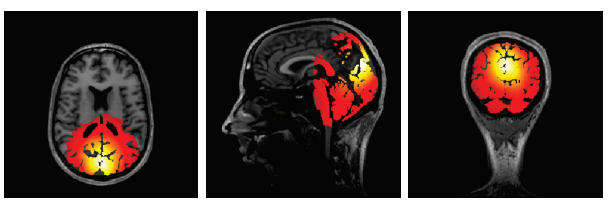

Step 2
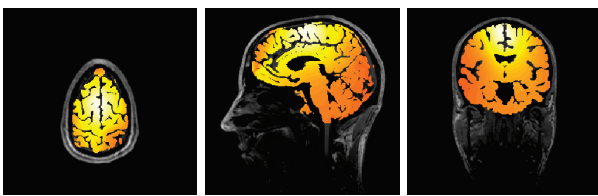

Step 3

Fig. 4: The output tomographic maps of MCSMB
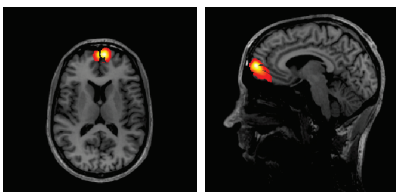

Step 1
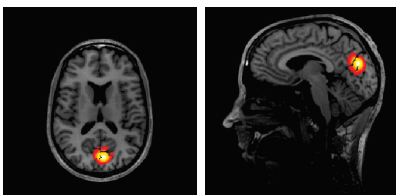

Step 2
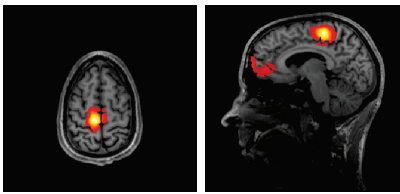

Step 3
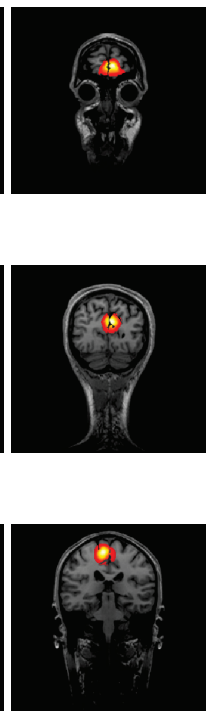

Fig. 5: The output tomographic maps of RC-MCSMB

beamformers are wide spread and the beamformers cannot correctly detect the 3 sources. We cannot use the prewhitening beamformer since we do not have background EEG data. Moreover, the prewhitening method would have a problem whenever background interferences and brain sources are highly correlated. The eigenspace approach is also only used to improve the quality of the reconstructed source, and not the localization. In fact it has been shown in [14] that for the single source case, the output of the eigenspace beamformers is a constant. The null-constrained beamformer cannot be used in this simulation since we do not have the interference sources. Further, there is not any framework to reconstruct the multiple correlated sources using the null-constrained beamformer.
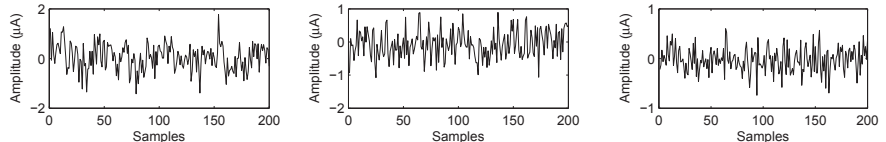

S-MVB
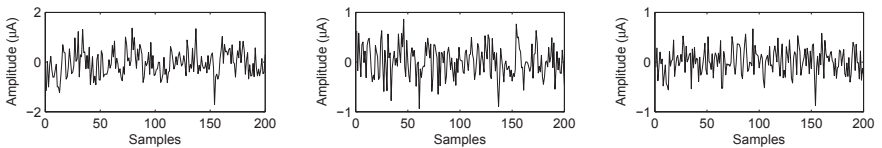

SAM
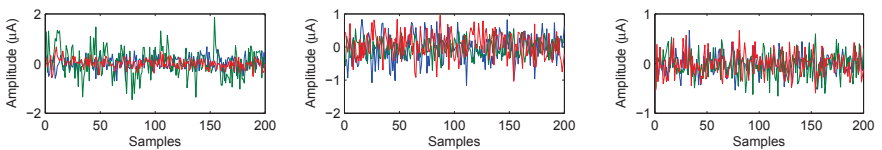

LCMV

Fig. 6: The 3 reconstructed source signals using S-MVB, $\mathrm{SAM}$, and LCMV with the input $S N R=4 \mathrm{~dB}$

According to Algorithm 1 in section 2.1, each correlated brain source needs to be identified separately using a recursive framework. Assuming the number of brain sources (NSOURCE) is known in advance, we break the whole process into NSOURCE steps. The tomographic map obtained from the $k^{t h}$ step is used to detect the $k^{t h}$ source, which is located at the global maximum in the map. In our simulation study, we have 3 simulated sources, thus 3 steps are required. The output tomographic maps obtained from these steps are given in Figure 4. By using MCSMB we can localize all 3 simulated brain sources with localization errors of $10.6 \mathrm{~mm}$, $5.1 \mathrm{~mm}$ and $24 \mathrm{~mm}$, respectively. As we can see, the output map of the third source is spread out because its power is much smaller than that of the first and second sources. The localization error of the third source is still large $(24 \mathrm{~mm})$. To reduce this localization error, we combine the RC and MCSM beamformers to form the Region Constrained-Multiple Correlated Source Model Beamformer (RC-MCSMB). Here, we assume the activated regions are known, given by spheres centered at the simulated source locations with a radius of 30 $\mathrm{mm}$. The steps described in Algorithm 1 are followed, with the covariance matrix $\mathbf{R}$ replaced by $\mathbf{R}+\lambda \mathbf{G}_{i} \mathbf{G}_{i}^{T}$, where $\mathbf{G}_{i}$ is the lead field matrix of the activation region, and the regularized parameter is given by $\lambda=\frac{2 \sigma_{\max }(\mathbf{R})}{\sigma_{\max }\left(\mathbf{G G}^{T}\right)}$, Using RC-MCSMB we can identify the three brain sources with corresponding localization errors of $4.6 \mathrm{~mm}, 1.7 \mathrm{~mm}$ and 1.4 $\mathrm{mm}$. The activated regions are shown in Figure 5.

In the source reconstruction study, we assume the locations of 3 brain sources are known. Once the EEG signals have been generated, we then use them to reconstruct the brain sources at their original locations. Figure 6 and 7 show the 3 reconstructed sources for the different beamformers studied. It can be seen very clearly that reconstructed sources of S-MVB, SAM and LCMV are very noisy and distorted, and their amplitudes are much smaller than those of the original sources. The output of MCSMB is similar to the 

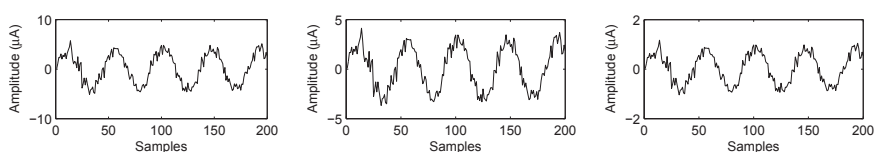

(a)
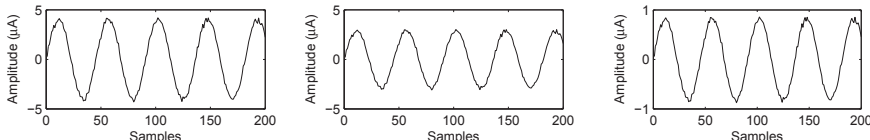

(b)

Fig. 7: The reconstructed sources using MCSMB (a) and eigen-MCSMB (b) with the input $S N R=4 \mathrm{~dB}$

original sources, however, they are still noisy. If we apply the eigenspace projection to MCSMB, then the reconstructed signals of this new beamformer are improved. However, the amplitudes of the reconstructed sources are reduced by about $20 \%$.

\section{CONCLUSIONS}

The region constrained beamformer is proposed which allows us to incorporate the knowledge of brain source regions into the beamformer design. The multiple correlated source model beamformer is also introduced. This beamformer can identify 3 perfectly correlated sources in the multiple source study. Most importantly, the combination of the region constrained and multiple correlated source model beamformers can identify 3 perfectly correlated brain sources with very high localization accuracy and reconstruction quality. This finding is significant as no beamformer has been able to identify more than 2 correlated sources with high localization accuracy in the past.

\section{REFERENCES}

[1] S. Baillet, J. C. Mosher, and R. M Leahy, "Electromagnetic brain mapping," Signal Processing Magazine, IEEE, vol. 18, no. 6, pp. 14-30, Nov 2001.

[2] B. D. Van Veen, W. Van Drongelen, M. Yuchtman, and A. Suzuki, "Localization of brain electrical activity via linearly constrained minimum variance spatial filtering," IEEE Trans. Biomed. Eng., vol. 44, pp. 867-880, 1997.

[3] S. E. Robinson and J. Vrba, "Functional neuroimaging by synthetic aperture magnetometry (SAM)," Recent Advances in Biomagnetism, pp. 302-305, 1999.

[4] K. Sekihara, S.S. Nagarajan, D. Poeppel, A. Marantz, and Y. Miyashita, "Reconstructing spatio-temporal activities of neural sources using an MEG vector beamformer technique," IEEE Trans. Biomed. Eng., vol. 48, pp. 760-771, 2001.

[5] J. Gross, J. Kujala, M. Hamalainen, L. Timmermann, A. Schnitzler, and R. Salmelin, "Dynamic imaging of coherent sources: Studying neural interactions in the human brain," PNAS, vol. 98, no. 2, pp. 694-699, January 2001.

[6] S.S. Dalal, K. Sekihara, and S.S. Nagarajan, "Modified beamformers for coherent source region suppression," IEEE Trans. Biomed. Eng., vol. 53, no. 7, pp. 1357 1363, July 2006.

[7] Matthew J. Brookes, Claire M. Stevenson, Gareth R. Barnes, Arjan Hillebrand, Michael I.G. Simpson, Susan T. Francis, and Peter G. Morris, "Beamformer reconstruction of correlated sources using a modified source model," Neuroimage, vol. 24, no. 3, pp. 14541465, 2007.

[8] K. Sekihara, S.S. Nagarajan, D. Poeppel, A. Marantz, and Y. Miyashita, "Asymptotic SNR of scalar and vector minimum-variance beamformers for neuromagnetic source reconstruction," IEEE Trans. Biomed. Eng., vol. 51, pp. 1726-1734, 2004.

[9] R.E. Greenblatt, A. Ossadtchi, and M.E. Pflieger, "Local linear estimators for the bioelectromagnetic inverse problem," IEEE Trans. Biomed. Eng., vol. 53, no. 9, pp. 3403-3412, Sept. 2005.

[10] K. Sekihara, S.S. Nagarajan, D. Poeppel, A. Marantz, and Y. Miyashita, "Performance of an MEG adaptivebeamformer technique in the presence of correlated neural activities: effects on signal intensity and time-course estimates," IEEE Trans. Biomed. Eng., vol. 49, pp. 1534-1546, 2002.

[11] Xiaohong Wan, Atsushi Sekiguchi, Satoru Yokoyama, Jorge Riera, and Ryuta Kawashima, "Electromagnetic Source Imaging: Backus-Gilbert Resolution Spread Function-Constrained and Functional MRI-Guided Spatial Filtering," Hum. Brain Mapp., vol. 29, no. 29, pp. 627-643, 2008.

[12] K. Sekihara, K.E. Hild, and S.S. Nagarajan, "A novel adaptive beamformer for MEG source reconstruction effective when large background brain activities exist," IEEE Trans. Biomed. Eng., vol. 53, no. 9, pp. 17551764, Sept. 2006.

[13] M. Diwakar, M-X Huang, R. Srinivasan, D. L. Harrington, A. Robb, A. Angeles, L. Muzzatti, R. Pakdaman, T. Song, R. J. Theilmann, and R. R. Lee, "Dual-core beamformer for obtaining highly correlated neuronal networks in MEG," Neurolmage, vol. In Press, Corrected Proof, pp. -, 2010.

[14] Hung V. Dang, "Performance analysis of EEG adaptive beamformers," M.S. thesis, New Mexico State University, 2007. 